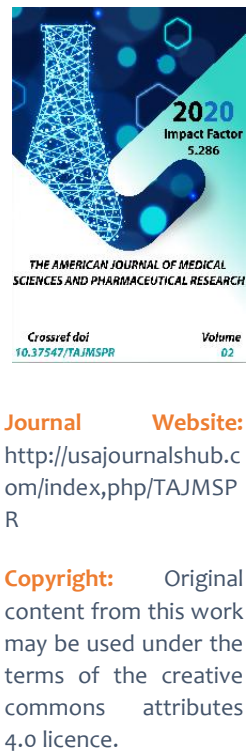

\title{
Complex Forecast Of The Consequences Of Secondary Encephalitis In Children
}

\author{
Niyazov Shukhrat Toshtemirovich, \\ Samarkand State Medical Institute, Samarkand, Uzbekistan \\ Djurabekova Aziza Takhirovna, \\ Samarkand State Medical Institute, Samarkand, Uzbekistan \\ Shomurodova Dilnoza Salimovna, \\ Samarkand State Medical Institute, Samarkand, Uzbekistan \\ Igamova Saodat Sur'atovna, \\ Samarkand State Medical Institute, Samarkand, Uzbekistan
}

\section{ABSTRACT}

The paper presents an analysis of a study of children with the consequence of secondary encephalitis, depending on the onset of the acute period of which more than 6 months - a year have passed, the degree of neurological disorders, neuroimaging comparison and prediction depending on these indicators for timely neurometabolic therapy are studied. The aim of our study was to study a comprehensive prognosis of the consequences of secondary encephalitis in children. There were 58 children under our supervision, with the consequence of the transferred encephalitis. The total number of girls is $37 \%$, boys are $63 \%$ of the main group of patients, the control group of children are healthy identical in age 3-10 years. All children admitted to the hospital underwent a standard examination, anamnesis, clinical neurological examination, and neuroimaging (MRI, CT) studies.

In addition, somatosensory evoked potential was determined for all children on electromyography, including at admission and also at discharge. The results of our study revealed that $66.2 \%$ gives predictions only for clinical and neurological disorders, with the addition of neuroimaging assessments it increased to $79 \%$, and meningeal-somatosensory indicators still increase the prognosis by $12 \%$, which is very important for timely neurometabolic therapy. In turn, the prognosis of the outcome of encephalitis in children will make it possible to promptly reduce residual neurological manifestations.

\section{KEYWORDS}

Secondary encephalitis, children, diagnosis, prognosis, therapy. 


\section{INTRODUCTION}

According to the WHO, a large percentage $(80 \%)$ of cases of central nervous system disorders, as a consequence of encephalitis, affects children. The nature of CNS damage in this pathology has a particular severity of neurological complications in childhood $[1,3]$. The degree of disability and mortality increased. This is due to the special structure of the blood-brain barrier, rather it is not before its development in children, which leads to necrotic disorders and the formation of a focus of decay of the medulla [4]. As a result, a cystic formation is formed, according to the literature, more in the temporal lobes. And the same sources provide data on the etiological factor, as a large percentage of encephalitis is usually the consequences of influenza in more than $50 \%$, the second place is taken by the consequences caused by the herpes virus $[4,5]$. Today the question is raised about childhood strokes caused by bacterial or viral encephalitis. Acute meningoencephalitis in children has high mortality rates, and as a consequence, gross disorders of the central nervous system develop [12].

An urgent issue in the problem of neuroinfections in children is predicting outcomes in the early stages of the disease, which will allow timely correction of therapy to reduce residual neurological disorders [9]. And an important component in this process, of course, is diagnostics. The severity of brain damage in encephalitis depends on several factors and the degree of damage to the central nervous system neurons in response to neuroinfection. Unambiguously, the method of neuroimaging ( $\mathrm{MRI}, \mathrm{CT}$ ) provides an assessment of the prevalence of inflammatory lesions of the brain structure, the severity of the process and allows tracking the dynamics of foci during the treatment period. But it does not allow assessing the functional state of the central nervous system in encephalitis, since electroencephalography [8]. According to Kepa L. (2000) EEG accurately predict the disease in $45 \%$ of cases, in addition, in the acute period, EEG data may be non-specific or even absent. According to the literature, the state of the conducting afferent systems of the brain and the state of the neuronal centers is possible by the method of determining the evoked potentials (EP). The same sources report on the use of $(E P)$ in neuroinfectious practice [6].

The aim is to study a comprehensive prognosis of the consequences of secondary encephalitis in children.

Materials and research methods. There were 58 children under our supervision, with the consequence of the transferred encephalitis. In the acute period, most children were treated at their place of residence, some $15 \%$ were in the children's general hospital in Samarkand, $5 \%$ in the emergency hospital in Samarkand, after 5-8 months (sometimes a year) in the children's neurological department of the 1st clinic SamMI. The research period is 2018-2020. The total number of girls is $37 \%$, boys are $63 \%$ of the main group of patients, the control group of children is healthy children, identical in age 3-10 years. All children admitted to the hospital underwent a standard examination, anamnesis, clinical neurological examination, and neuroimaging (MRI, CT) studies. Children of the main group are divided into two subgroups, subgroup 1 - 18 children, children who have had 6 months from the onset of the acute period, subgroup 2 - 40 children, 
children with the disease more than 6 months - 1 year. Along with neuroclinical and neuroimaging examinations, somatosensory evoked potential was determined for all children on electromyography, including at admission and at discharge. The norm was taken as an amplitude indicator of 3-5 $\mu \mathrm{V}$ of the cortical potential, calculation of the central afferent conduction depending on age. Statistical data processing of analysis methods according to Spearman, assessment of reliability according to Fikkr.

Research results. Examination of sick children revealed focal neurological symptoms, expressed by various syndromes. There are no distinct meningeal symptoms, but decerebral rigidity was noted in 39\% of cases. Among cranial changes, the percentage of amauroses is high, children most often do not fix their gaze, examination by an ophthalmologist confirms atrophy of the optic nerves in $23.8 \%$, paresis of the gaze was noted in 2 patients, examination is limited due to lack of contact with the patient, in all children loss acquired skills, delayed psychoverbal development in $100 \%$ of cases. In our opinion, the violation of swallowing according to the pseudobulbar type was severe, the children kept food in their mouth for a long time, they could swallow only in liquid form, symptoms of dysphagia were $50 \%$ on the face, paresis of the seventh (VII) pair was noted in $49.1 \%$, sensorineural hearing loss III- IV degree (in patients in infancy, it was not possible to conduct audiometry, it was carried out in older children). Persistent movement disorders were manifested in the form of central spastic tetraparesis in $38.8 \%$, hemiparesis to a lesser extent in $31.3 \%$. In other cases, there were mixed symptoms of tetraparesis with hyperkinetic syndrome, or tetraparesis with atactohyperinetic syndrome. Hypotonic-hyperkinetic syndrome was observed in 4 children, atotic astatic syndrome in 5 patients. In the examined patients, there is a sharp increase in tendon reflexes on the pasty side, in the same areas, pathological reflexes are clearly visible. The pathological side had a high tone, not rough but noticeable muscle atrophy on the side of paresis, contracture of the limbs was noted in $60.2 \%$. This was especially noticeable in spastic tetraparesis, the legs crossed at any attempt to touch the limbs. Clonus was noted in $25.5 \%$, a protective reflex in $16.9 \%$ of cases.

Sensitive disorders cannot be traced in such patients due to reduced intelligence and age category, but more often children reacted to injection procedures with crying and motor restlessness. Since the children in the group of our examination were in a residual position, we tried to determine the level of impairment of higher cortical functions. A pronounced delay in psychoverbal development was revealed in $40 \%$ of children (these were mainly children with tetraparesis), dementia in $11 \%$ of cases, the average level of delay was $9.1 \%$, epileptic seizures were noted in $12.6 \%$ of cases, symptomatic epilepsy $78.5 \%$ and the epileptic seizures themselves were manifested as partial or secondary generalized seizures.

An important and reliable diagnostic method for patients with the consequence of encephalitis and meningoencephalitis is neuroimaging MRI / CT examination. In 23.3\%, the outcome of encephalitis was occlusive hydrocephalus, in $78.7 \%$ there was cerebral atrophy, and in $55.7 \%$ of hydrocephalus, varying degrees of ventriculodelation in 9 children, secondary pairs of encephalic cysts in $8.5 \%$, in 3 patients revealed a chronic subdural hematoma. In $15 \%$ of cases, pronounced cysticgliatic transformation was noted in both hemispheres. Expansion of CSF spaces in 7.5\% of cases. 


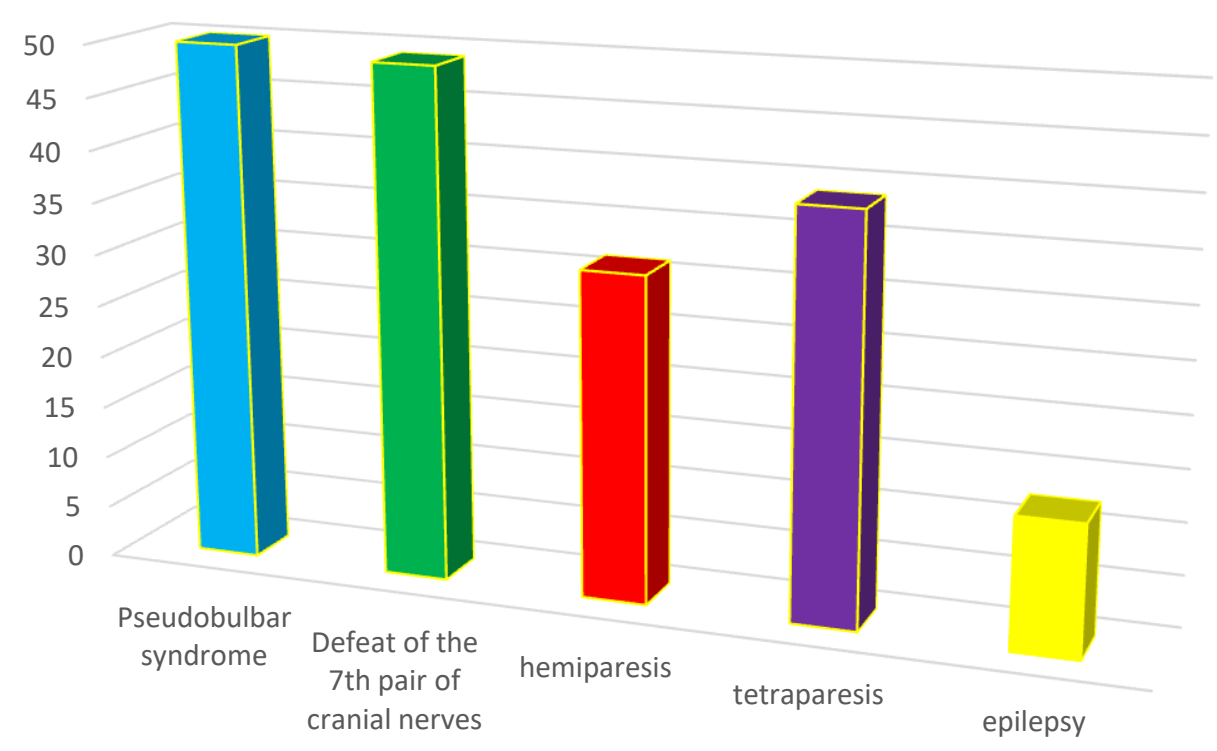

Table 1.

Ratio of predicted and actual outcomes of encephalitis $(n=58)$

\begin{tabular}{|c|c|c|c|}
\hline Outcome & $\begin{array}{c}\text { Correctly diagnosed } \\
\text { by the proposed } \\
\text { method of abs. } \\
\text { amount }(\%)\end{array}$ & $\begin{array}{c}\text { False positive }(*) \text { and false } \\
\text { negative }(* *) \text { results, abs. } \\
\text { amount }(\%)\end{array}$ & $\begin{array}{c}\text { Actual outcomes } \\
\text { of the disease, } \\
\text { abs. amount }(\%)\end{array}$ \\
\hline $\begin{array}{c}\text { Favorableoutcome } \\
(*)\end{array}$ & $33 \%$ & $2 \%$ & $42 \%$ \\
\hline $\begin{array}{c}\text { Unfavorableoutcome } \\
(* *)\end{array}$ & $48 \%$ & $28 \%$ & $528 \%$ \\
\hline
\end{tabular}

\section{Table 2.}

Diagnostic efficiency of various methods for predicting the outcomes of viral encephalitis in children $(n=58)$

\begin{tabular}{|c|c|c|c|c|}
\hline & $\begin{array}{c}\text { Clinicalandneurolo } \\
\text { gicaldisorders (\%) }\end{array}$ & $\begin{array}{c}\text { MRI } \\
\text { abnormalitie } \\
\mathrm{S}(\%)\end{array}$ & $\begin{array}{c}\text { Clinicand } \\
\text { MRI (\%) }\end{array}$ & $\begin{array}{c}\text { Comprehensive } \\
\text { analysis of clinical } \\
\text { neurological, MRI } \\
\text { and functional } \\
\text { disorders (\%) }\end{array}$ \\
\hline
\end{tabular}




\begin{tabular}{|c|c|c|c|c|}
\hline Specificity & 90,0 & 75,5 & 79,1 & 92,2 \\
\hline Sensitivity & 43,0 & 22,0 & 49,0 & 64,4 \\
\hline Efficiency & 66,2 & 68,5 & 79,0 & 85,8 \\
\hline
\end{tabular}

The effectiveness of using the method for studying somatosensory potentials showed more than 90\% reliability, especially in predicting a favorable outcome of encephalitis.

According to these criteria, the outcome of encephalitis was favorable; it was predicted in children with a consequence of neuroinfection during the first 6 months. According to the prognosis scale, these are children with moderate neurological symptoms, with 1-2 foci on MRI, the cortical potential being higher than $3 \mu \mathrm{V}$, the indicator of which increases by $15 \%$ from the initial values. An unfavorable outcome is observed in children with focal disorders, the duration of which prevails 8-12 months from the onset of the disease, in such children there are residual focal neurological manifestations of convulsive syndrome, MRI revealed more than 3-5 foci, a decrease in the amplitude of the cortical potential below $3 \mu \mathrm{V}$ and, accordingly, negative dynamics ...

Complete recovery was not observed, positive dynamics with partial disappearance of neurological symptoms were observed in $23 \%$ of children observed from the onset of the disease, in whom 6 months passed, that is, 1 subgroup. An unfavorable outcome was observed in children of the 2nd subgroup of patients with more than 6 months, 1 year, persistent focal neurological disorders were noted in $55 \%$, residual effects in $25 \%$ of cases were convulsive syndrome. It was predicted only for clinical and neurological disorders gives $66.2 \%$, with the addition of neuroimaging assessment, it increased to $79 \%$, and meningeal-somatosensory indicators still increase the prognosis by $12 \%$, which is very important for timely neurometabolic therapy.

\section{CONCLUSIONS}

1. Children with a consequence of encephalitis need a comprehensive assessment of the severity state, taking into account the child's age, clinical and neurological disorders, MRI data and somatosensory evoked potentials, for the correct selected correction of neurometabolic therapy.

2. The prognosis of the outcome of encephalitis in children will allow timely reduction of residual neurological manifestations.

\section{REFERENCES}

1. Bondarenko A.L. Neurological manifestations of chronic Lyme borreliosis. // Journal of Infectious Diseases. - No. 3 - T-4 - 2016. P. 60-63

2. Gladkiy P. A., Sergeeva I. G., Tulupov A. A. Infectious lesions of the brain. Study guide - Novosibirsk 2015

3. Gusev E.I. Boyko A.N. Demyelinating diseases of the central nervous system. Consilium-Medicum, 2009. - T.2. -№2. - S. 96-120

4. Mironenko T.V., Pogorelova G.A. Viral encephalitis, issues of diagnosis and treatment. // Journal of the Ukrainian Medical Almanac. -№ 2 - Volume 15 - 2012.

5. Olenkova O.M. Clinical and immunological features of the meningeal form of enterovirus infection (echo - and coxsackie in - viral etiology) in children. Diss. Ph.D. - Yekaterinburg 2015

6. Savina M.V. The role of evoked brain potentials in predicting the course and outcomes of encephalitis in children. Diss. Ph.D. - St. Petersburg 2009 
7. Skripchenko N.V. Neuroinfection in children in modern conditions. // Journal of Practical Medicine. - 10 (111) December 2017 - pp. 7-15

8. StarshinovYa. Yu. Clinical and radiation and immunological features of encephalomyelitis in children: author. dis. ... Ph.D. - SPb., 2003 -- 23 p.

9. Khmara M.E. Comparative clinical and morphological characteristics of acute and chronic herpetic encephalitis. -Clinical Department of Neuroviral Infections GU NIIEM MH RB - 2018

10. Childhood encephalitis in Sweden: Etiology, clinical presentation and outcome / A. Fowler, A. Stockberg, M. Eriksson, R. Wickstrom // Eur. J. Paediatr. Neurol. - 2008. - V. 9. - No. 4. - P. 129-135.

11. Kalita J. Neurophysiological changes in Japanese encephalitis / J. Kalita, U. Misra // Neurol. India. - 2002. - V. 50. - No. 2. - P. 262-266. 